\title{
Correlations Among Subcutaneous Immunoglobulin Dosage, Immunoglobulin G Serum Pre-infusional Levels and Body Mass Index in Primary Antibody Deficiency Patients: A Pooled Analysis from the SHIFT/IBIS Studies
}

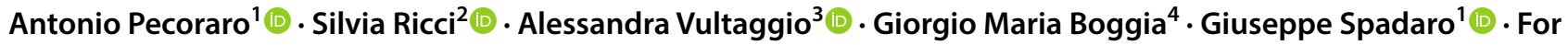 \\ the SHIFT and IBIS Study Groups
}

Published online: 8 February 2020

(c) The Author(s) 2020

\begin{abstract}
Background and Objective In recent years, two Italian non-interventional studies evaluated subcutaneous immunoglobulin (SCIG) treatment in patients affected by primary antibody deficiency (PAD). The SHIFT study considered patients who were treated with intravenous immunoglobulin (IVIG) or SCIG 16\% (Vivaglobin ${ }^{\circledR}$ ) and then replaced this therapy with weekly treatments of SCIG 20\% (Hizentra $^{\circledR}$ ). The IBIS study evaluated patients previously taking a weekly SCIG 20\% regimen, who instead began therapy with biweekly SCIG $20 \%$ to assess the correlation between the dose of immunoglobulin G (IgG) administered and the body mass index (BMI) of patients, determine if there is a need for dosage adjustments on a BMI basis, and identify the predictors of serum $\operatorname{IgG}$ trough levels in our cohort.

Methods In this study, we analyzed the pooled data of 109 PAD patients enrolled in the SHIFT and IBIS studies. Only prospective phases were considered.

Results The total monthly SCIG dose showed comparable trends among weight categories, except for underweight patients. When we considered the monthly SCIG dosage per kilogram of body weight, a significant decreasing trend according to BMI was observed. Data on IgG trough levels were available for 88 patients, with a mean IgG serum level of $8.4 \pm 1.6 \mathrm{~g} / \mathrm{L}$. A stepwise regression model revealed that the mean monthly dosage of SCIG 20\% ( $p=0.04248)$ and the mean monthly dosage of IgG per kilogram of body weight were the only two independent predictors associated with IgG trough levels. No association was found between BMI and IgG trough levels.

Conclusions These findings support the concept that the cumulative monthly dose of SCIG and the dose of SCIG per kilogram of body weight affect IgG trough levels in PAD patients, irrespective of BMI.
\end{abstract}

\section{Key Points}

Giuseppe Spadaro

spadaro@unina.it

1 Department of Translational Medical Sciences and Center for Basic and Clinical Immunology Research (CISI), University of Naples Federico II, Via S. Pansini 5, 80131 Naples, Italy

2 Division of Pediatric Immunology, Department of Health Sciences, University of Florence and Meyer Children's Hospital, Viale G. Pieraccini 24, 50139 Florence, Italy

3 Immunoallergology Unit, Department of Biomedicine, Azienda Ospedaliero-Universitaria Careggi, Largo Brambilla 3, 50134 Florence, Italy

4 Medical Affairs, CSL Behring, V.le del Ghisallo 20, 20151 Milan, Italy
Data for 109 patients from two Italian observational studies (SHIFT and IBIS) who were affected by primary antibody deficiency (PAD) were pooled to investigate the presence of possible correlations among pre-infusion concentration, immunoglobulin $\mathrm{G}$ ( $\mathrm{IgG})$ dosage, and body mass index (BMI).

BMI did not correlate with trough concentrations, and IgG dosage had comparable trends among weight categories (except for underweight patients).

IgG dose adjustment seems to be unnecessary in overweight and obese patients. 


\section{Introduction}

Primary antibody deficiency (PAD) includes a heterogeneous group of disorders affecting one or more functions of the immune system [1]. With the growing development of genetic equipment, many new genes affected, and their related mutations, have been discovered. PADs encompass a myriad of presentations, ranging from immune deficitrelated symptoms (i.e. infections and malignancies) and immune dysregulation-related manifestations (i.e. autoinflammation phenomena, autoimmunity disorders and allergies) [2]. PADs have been estimated to affect 6 million people worldwide [3]. According to the European Society for Immunodeficiency Database (ESID), more than $50 \%$ of the registered cases of PADs are "predominantly antibody disorders" [4].

The gold-standard treatment for patients with PAD is immunoglobulin $\mathrm{G}$ (IgG) replacement therapy (IGRT) [5]. Intravenous (IVIG) and subcutaneous (SCIG) infusions are the two routes used to administer IgG. In contrast to IVIG, SCIG allows home-based self-administration and results in more stable IgG serum levels, thus improving the quality of life and clinical efficacy. In addition, the self-administration of IgG improves patient compliance and empowerment [6]. Most national and international guidelines suggest a mean dosage of $0.4-0.6 \mathrm{~g} / \mathrm{kg} / \mathrm{month}$ for both IVIG and SCIG [7]; however, according to the Hizentra ${ }^{\circledR}$ Summary of Product Characteristics (SPC), IgG dosage needs to be adjusted on an individual basis, with the aim of reaching $\mathrm{IgG}$ trough levels $>5-6 \mathrm{~g} / \mathrm{L}$.

Two Italian observational studies, SHIFT [8] (CSL Behring protocol IgPro20_5001) and IBIS [9] (CSL Behring protocol IgPro20_5002), analyzed PAD patients requiring IGRT. In the SHIFT study, patients who were previously receiving therapy with IVIG or weekly SCIG $16 \%$ (Vivaglobin ${ }^{\circledR}$; CSL Behring, King of Prussia, PA, USA) were then treated with weekly SCIG 20\% (Hizentra ${ }^{\circledR}$; CSL Behring), while the IBIS study evaluated the real-life aspects of patients taking weekly Hizentra ${ }^{\circledR}$ who were then administered biweekly (i.e. every other week) treatments with Hizentra ${ }^{\circledR}$. Both studies showed that mean serum IgG trough levels were maintained above the protective threshold of $5 \mathrm{~g} / \mathrm{L}$.

This analysis pooled data from the SHIFT and IBIS studies to (1) assess the correlation between the dose of SCIG administered and the body mass index (BMI) of patients; (2) determine if there is a need for dosage adjustments on a BMI basis; and (3) identify the predictors of IgG trough levels in our cohort.

\section{Methods}

Patient data on the prospective phases from IBIS and SHIFT were pooled and uniformed in a unique database.

\subsection{Monthly Dosage: Assumptions}

For patients enrolled in the SHIFT study, monthly dosage was retrieved from the screening visit, since all patients were deemed stabilized by their treating physician at enrollment, and the dosages registered in the 3-and 6-month visits were reported as summary statistics without any difference from baseline. Therefore, we assumed that variations in the dosage during the study were negligible.

For patients enrolled in the IBIS study, the monthly dosage was calculated starting from the mean dose per infusion and the infusion frequency (number of days between two consecutive infusions).

\subsection{Body Mass Index-Based Categorization of Patients}

Adult patients were categorized according to the World Health Organization (WHO) criteria [10] based on BMI calculation (the weight of an individual in kilograms divided by the square of the height of an individual in meters), i.e. underweight: below $18.5 \mathrm{~kg} / \mathrm{m}^{2}$; normal weight: in the $18.5-24.9 \mathrm{~kg} / \mathrm{m}^{2}$ range; overweight or pre-obese: in the $25.0-29.9 \mathrm{~kg} / \mathrm{m}^{2}$ range; and obese: over $30 \mathrm{~kg} / \mathrm{m}^{2}$.

In contrast, as reported by the WHO [11], pediatric patients have to be evaluated on the basis of percentile tables, as BMI in patients under 18 years of age is characterized by high variability and is mainly sex- and agerelated. Therefore, pediatric patients were categorized as underweight if BMI was less than the 5th percentile, normal weight if BMI was in the 5th-85th percentile range, overweight if BMI was in the 86th-97th percentile range, and obese if BMI was greater than the 97 th percentile.

However, we used the BMI-for-age clinical growth charts of Cacciari and colleagues [12], which are similar to those of the WHO and are more representative of the Italian population. As in the latter curves, data regarding the 5 th percentile were not available, and we used the 3 rd percentile as a proxy.

\subsection{Statistical Analyses}

The association among categorical variables was measured using the Chi square test. Analysis of variance (ANOVA) was used to evaluate the mean differences among groups. Statistical analyses and the relevant graphics were performed using the statistical software R [13]. 
To evaluate the influence of all the possible predictors on the serum IgG trough concentration, a linear regression analysis was performed considering all the independent variables available in the database: administration frequency, type of patient (adult or pediatric), age, sex, height, weight, BMI, weight category (underweight, normal weight, overweight, obese), and monthly dosage (total and per kilogram of body weight). The model was analyzed using the stepwise regression technique to determine the best regression model with the lowest number of variables.

\section{Results}

A pooled database containing 111 patients was obtained from aggregation of the SHIFT (76 patients) and IBIS (35 patients) studies. Two patients from the SHIFT study were excluded as data regarding monthly dosage was not available, leaving 109 patients to be analyzed. Table 1 shows the general characteristics of the pooled database.

The populations of the two databases were comparable in all characteristics except age; IBIS patients were, on average, 8 years younger than SHIFT patients $(p=0.0152)$.
Analysis of the pediatric cohort included in the prospective phases of the SHIFT and IBIS studies was performed by Moschese et al. and is under consideration for publication.

In the pooled database analyzed in this study, $60 \%$ of patients were normal weight, $35 \%$ were overweight or obese, and 5\% were underweight. No statistically significant differences among the distribution of weight categories were detected when analyzing adult and pediatric populations separately (Chi square test, $p=0.2022$ ).

The distribution of weight categories in the adult population is comparable with that observed in the Italian population aged $>18$ years [14] (Fig. 1a). In contrast, the distribution of weight categories in the pediatric population is slightly different from the national mean [15], particularly in the overweight category (Fig. 1b).

\subsection{Immunoglobulin G Dosage and Pre-infusion Trough Concentration}

Total monthly IgG dosage showed comparable trends among weight categories, except for underweight patients (Table 2, Fig. 2a), as highlighted by the ANOVA test $(p=0.02428)$.

Table 1 General characteristics of the pooled database, aggregating the SHIFT (74 patients) and IBIS (35 patients) databases

\begin{tabular}{|c|c|c|c|c|c|}
\hline Characteristic & $N(\%)$ & Mean (SD) & Median (IQR) & Range & $p$ value \\
\hline Sex, male & $64(58.7)$ & & & & 0.2191 \\
\hline SHIFT & $40(54.1)$ & & & & \\
\hline IBIS & $24(68.6)$ & & & & \\
\hline Age, years & & $31.4(16.3)$ & $29(18-43)$ & $2-71$ & 0.0152 \\
\hline SHIFT & & $33.9(16.7)$ & $33(21-47)$ & $2-71$ & \\
\hline IBIS & & $26.1(14.4)$ & $24(14-40)$ & $2-56$ & \\
\hline Height, $\mathrm{cm}$ & & $163.9(18.2)$ & $168(158-175)$ & $87-191$ & 0.3313 \\
\hline SHIFT & & $165.1(17.5)$ & $169(158-177)$ & $87-190$ & \\
\hline IBIS & & $161.5(19.5)$ & $166(157-174)$ & 87-191 & \\
\hline Weight, $\mathrm{kg}$ & & $64.8(19.1)$ & $65(52-79)$ & $12-100$ & 0.3022 \\
\hline SHIFT & & $66.2(18.9)$ & $66(55-85)$ & $12-100$ & \\
\hline IBIS & & $62.1(19.4)$ & $65(50-78)$ & $12-91$ & \\
\hline BMI, $\mathrm{kg} / \mathrm{m}^{2}$ & & $23.5(4.5)$ & $23(20-26)$ & $14-35$ & 0.5396 \\
\hline SHIFT & & $23.7(4.4)$ & $23(21-27)$ & $14-35$ & \\
\hline IBIS & & $23.1(4.7)$ & $24(20-27)$ & $14-34$ & \\
\hline Monthly IgG dosage, g & & $23.0(9.2)$ & $24(16-26)$ & $4-50$ & 0.5899 \\
\hline SHIFT & & $24.0(9.6)$ & $24(16-27)$ & $4-50$ & \\
\hline IBIS & & $21.0(8.1)$ & $22(16-26)$ & $5-35$ & \\
\hline Monthly $\mathrm{IgG}$ dosage, $\mathrm{mg} / \mathrm{kg}$ & & $365.5(132.1)$ & $357(268-414)$ & $152-33$ & 0.9199 \\
\hline SHIFT & & $374.9(143.8)$ & $357(276-405)$ & $194-833$ & \\
\hline IBIS & & $345.5(102.0)$ & $364(258-432)$ & $152-521$ & \\
\hline Pediatric patients & $28(25.7)$ & & & & 0.0994 \\
\hline SHIFT & $15(20.3)$ & & & & \\
\hline IBIS & $13(37.1)$ & & & & \\
\hline
\end{tabular}

$B M I$ body mass index, $I Q R$ interquartile range, $S D$ standard deviation, $I g G$ immunoglobulin 
When underweight patients $(n=5)$ were excluded, an homogeneous dosage was obtained among normal weight, overweight, and obese patients $(p=0.1092)$. In the monthly IgG dosage per kilogram of body weight, a significant decreasing trend according to BMI was observed (Table 2, Fig. 2b). These differences among groups were significant, independent of the inclusion or exclusion of the underweight category ( $p=0.04066$ and $p=0.03673$, respectively).

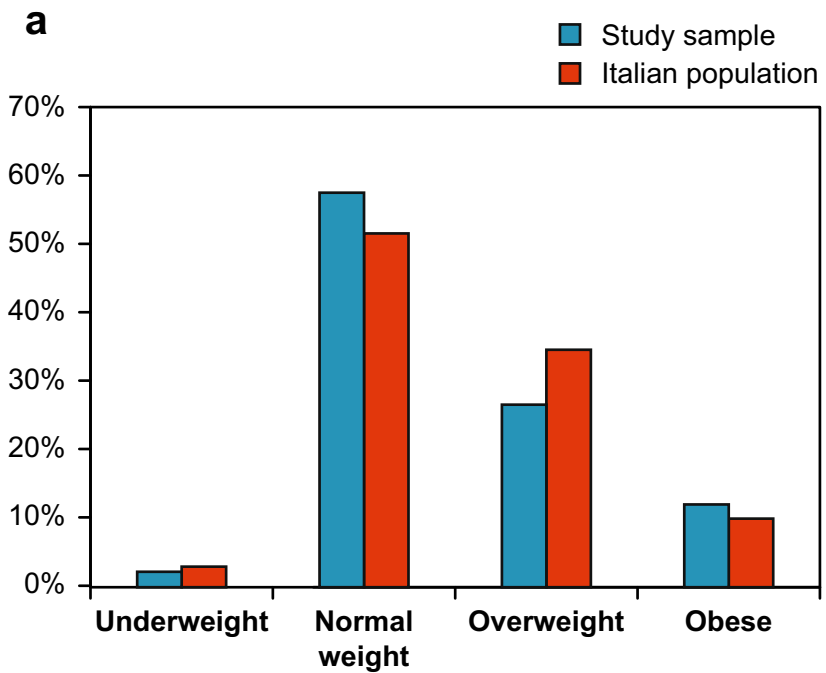

Fig. 1 a Study sample and the Italian adult population are compared in terms of distribution of weight categories, showing similar results. b Study sample and the Italian pediatric population are compared in
Data on the pre-infusion trough concentrations were available for 88 patients (Table 2 ). The mean pre-infusion trough concentration was $8.4 \mathrm{~g} / \mathrm{L}$, and for every patient it was $>5 \mathrm{~g} / \mathrm{L}$ (range $5.5-13.9 \mathrm{~g} / \mathrm{L}$ ). This predictor was comparable in the weight categories $(p=0.5331)$ (Fig. 3), even though a non-significant lower mean was detected in the obese group $(7.8 \mathrm{~g} / \mathrm{L})$.

A stepwise regression was performed to highlight factors predicting serum IgG trough levels (Fig. 4). The
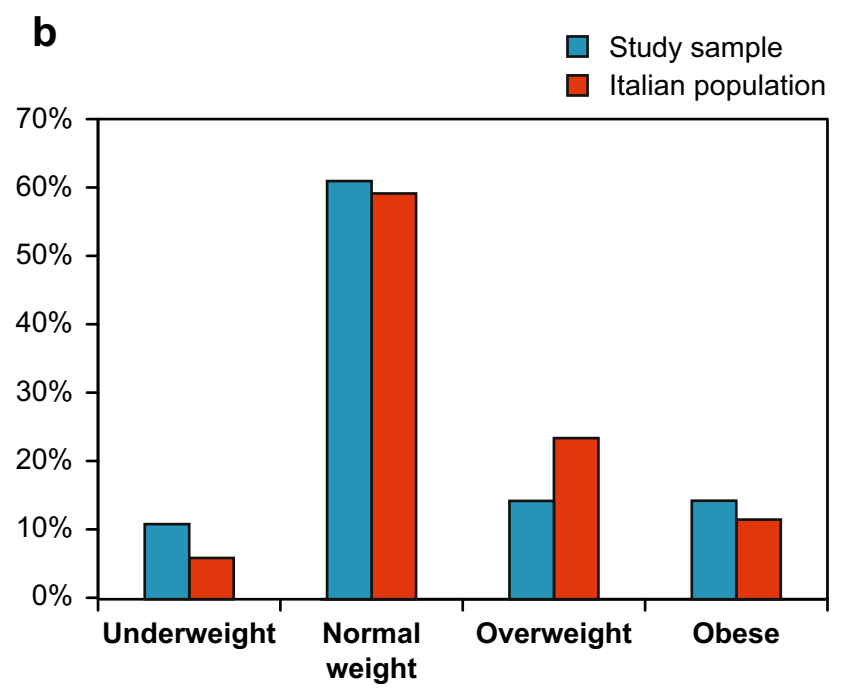

terms of the distribution of weight categories, showing slight differences, especially in the overweight category

Table 2 IgG monthly dosage administered (total and per kilogram of body weight) per weight category and pre-infusion trough serum levels (g/L; total and per weight category)

\begin{tabular}{|c|c|c|c|c|}
\hline Variable & $N(\%)$ & Mean (SD) & Median (IQR) & Range \\
\hline IgG dosage, $\mathrm{g} / \mathrm{month}$ & $109(100)$ & $23.0(9.2)$ & $24(16-26)$ & $4-50$ \\
\hline Underweight & $5(4.6)$ & $14.2(4.2)$ & $16(10-16)$ & $10-19$ \\
\hline Normal weight & $64(58.7)$ & $22.1(8.1)$ & $24(16-24)$ & $4-50$ \\
\hline Overweight & $26(23.9)$ & $26.5(11.1)$ & $22(17-35)$ & $12-50$ \\
\hline Obese & $14(12.8)$ & $23.9(9.0)$ & $24(21-30)$ & $6-40$ \\
\hline IgG dosage, $\mathrm{mg} / \mathrm{kg} / \mathrm{month}$ & $109(100)$ & $365.5(132.1)$ & $357(268-414)$ & $152-833$ \\
\hline Underweight & $5(4.6)$ & $437.0(95.1)$ & $406(400-457)$ & $334-588$ \\
\hline Normal weight & $64(58.7)$ & $385.1(132.6)$ & $374(304-422)$ & $166-833$ \\
\hline Overweight & $26(23.9)$ & $344.3(147.6)$ & $285(231-409)$ & 195-794 \\
\hline Obese & $14(12.8)$ & $289.7(64.4)$ & $284(259-330)$ & $152-412$ \\
\hline $\begin{array}{l}\text { Pre-infusion trough IgG concentration, } \\
\text { g/L }\end{array}$ & $88(80.7)$ & 8.4 (1.6) & $8.1(7.1-9.5)$ & $5.5-13.9$ \\
\hline Underweight & $5(5.7)$ & $8.4(2.5)$ & $8.2(6.6-10.8)$ & $5.5-11.1$ \\
\hline Normal weight & $50(56.8)$ & $8.5(1.5)$ & $8.3(7.4-9.7)$ & $6.2-13.9$ \\
\hline Overweight & $21(23.9)$ & $8.3(1.8)$ & $7.8(7.0-9.4)$ & $6.0-13.0$ \\
\hline Obese & $12(13.6)$ & $7.8(1.5)$ & $7.4(6.5-8.2)$ & $6.2-11.5$ \\
\hline
\end{tabular}

$I Q R$ interquartile range, $S D$ standard deviation, $I g G$ immunoglobulin $\mathrm{G}$ 


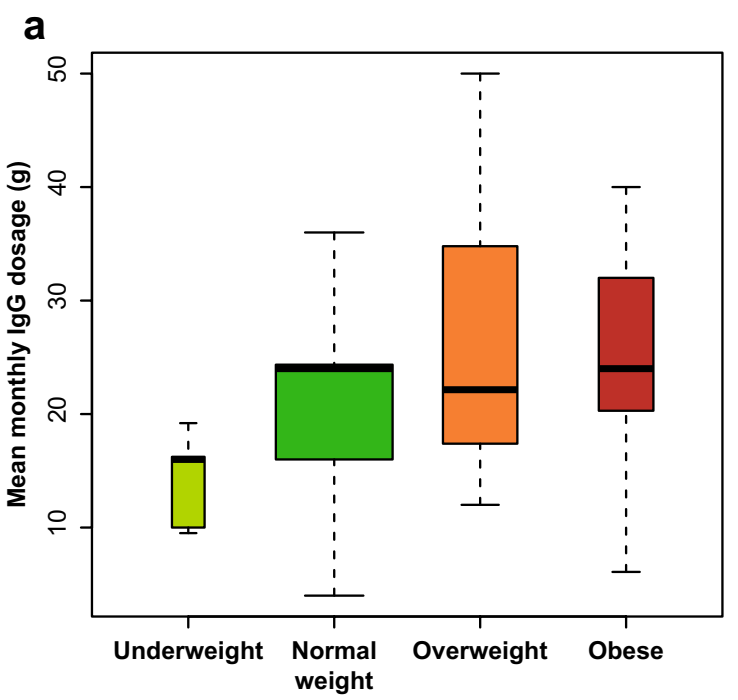

Fig. 2 a Total monthly IgG dosage per weight category. b Monthly IgG dosage per kilogram according to weight categories. The bold lines represent the median values, the boxes indicate the interquartile

mean monthly dosage of SCIG $20 \%(p=0.04248)$ and the mean monthly dosage of IgG per kilogram of body weight $(p=0.03312)$ were the only two independent predictors associated with pre-infusion IgG serum concentrations. These relationships, even if significant, were weakly linear; the model had a very low adjusted $R^{2}(0.03592$ and 0.04068 , respectively).

BMI was eliminated in the regression model during the selection of the variables. Figure $4 \mathrm{c}$ shows that this variable did not correlate with the pre-infusion trough concentration.

\section{Discussion}

International guidelines recommend dose immunoglobulin replacement therapy with reference to actual body weight [7]; however, how dosing might be tailored to maximize efficacy while minimizing costs is an open question. From this perspective, pinpointing the factors that mainly affect $\mathrm{IgG}$ trough levels in each individual patient is a very important issue. Over the past decades, several studies have investigated the efficacy, safety, tolerability, and pharmacokinetics of various novel IVIG or SCIG preparations [16-19]; however, the main outcomes of these studies were the rates of serious bacterial infections and drug-related adverse events rather than the definition of the most correct doses to achieve a suitable IgG trough level.

In our retrospective analysis of the pooled data derived from the SHIFT and IBIS studies, the mean monthly dosage of SCIG $20 \%$ and IgG per kilogram of body weight were the only two independent predictors associated

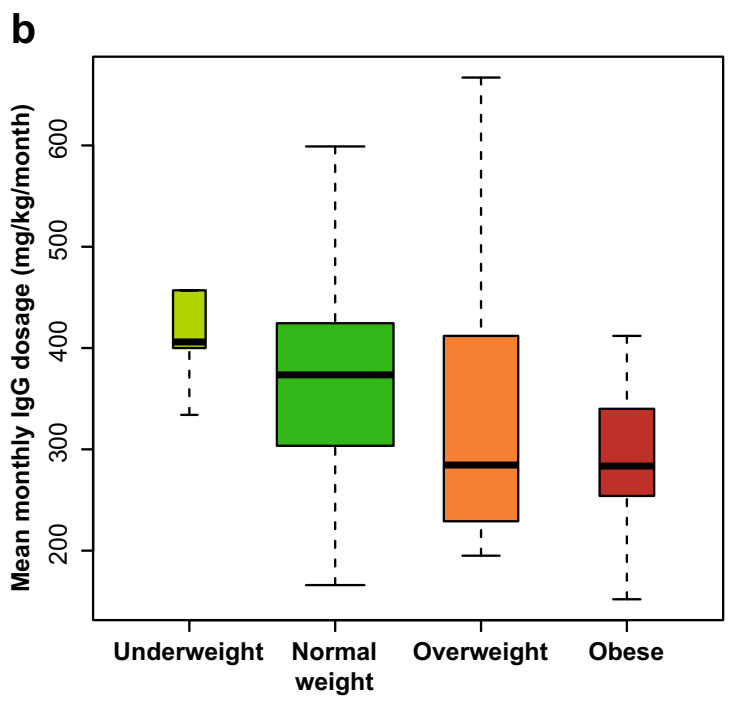

range, and the whiskers represent the minimum and maximum values. $B M I$ body mass index, $\operatorname{Ig} G$ immunoglobulin $\mathrm{G}$

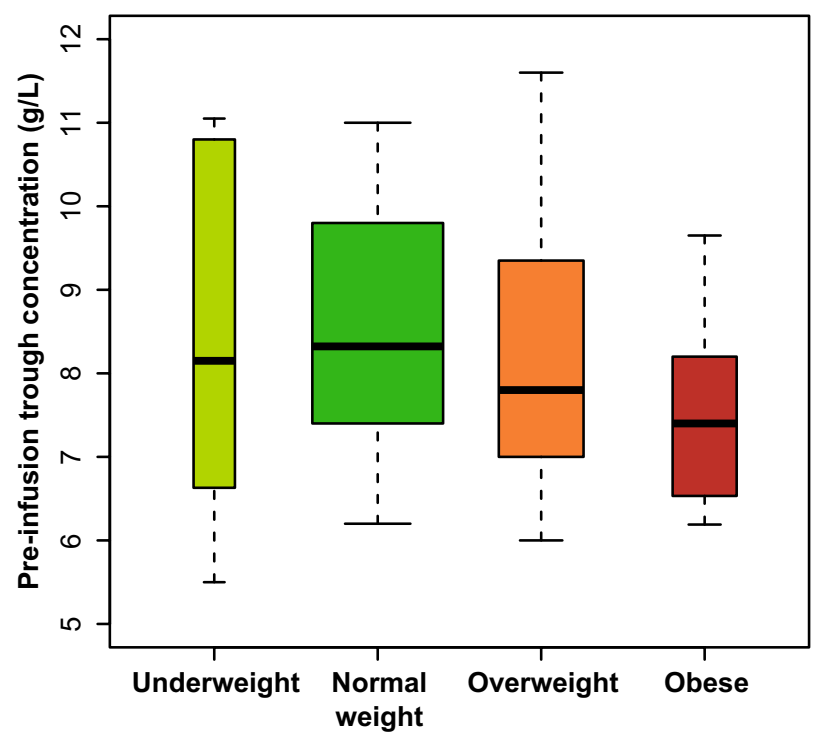

Fig. 3 Pre-infusion trough concentration $(\mathrm{g} / \mathrm{L})$ per weight category. The bold lines represent the median values, the boxes indicate the interquartile range, and the whiskers represent the minimum and maximum values

with IgG trough levels in patients treated with IVIG or SCIG 16\% immunoglobulin replacement therapy who subsequently replaced that therapy with SCIG $20 \%$. On the other hand, no association was found between BMI and IgG trough levels. These findings are consistent with the results of a previous large cohort study involving 40 obese PAD patients [20]. In particular, similar SCIG dose/ serum IgG level ratios were observed between obese and 
a

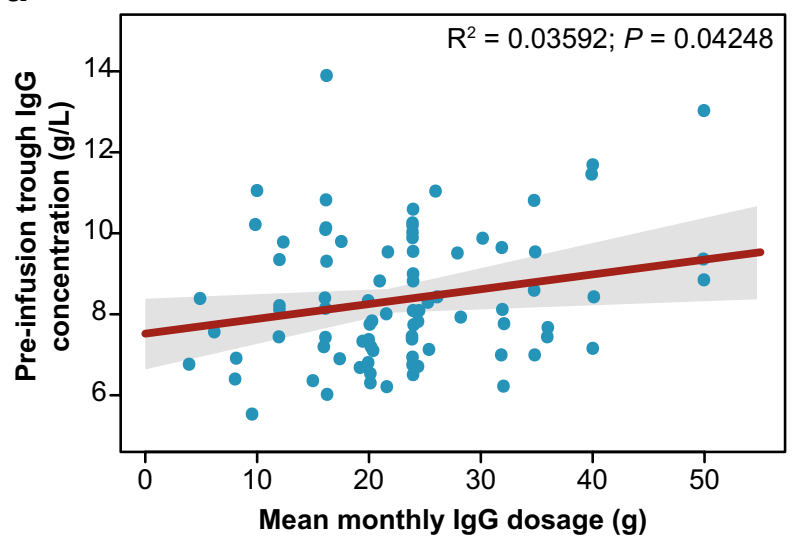

b

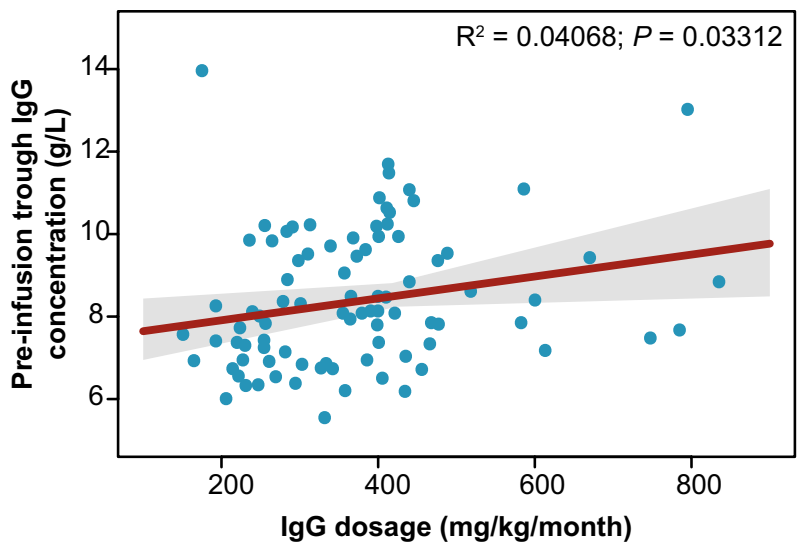

C

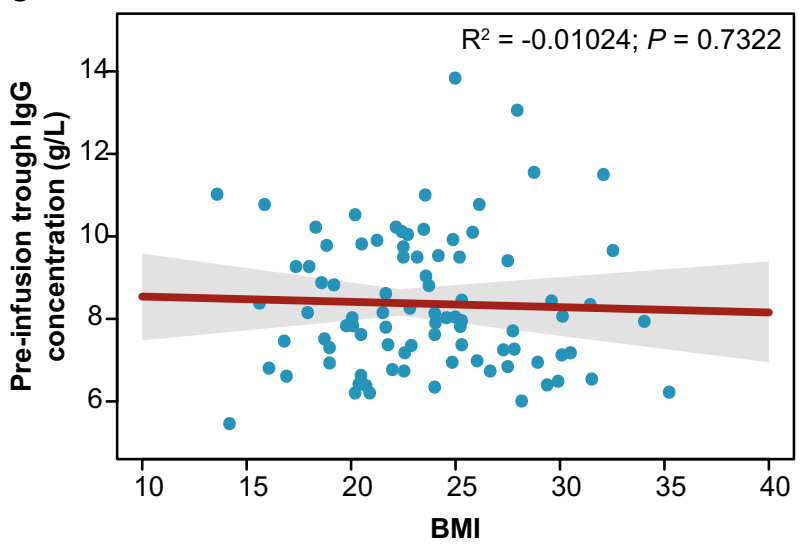

Fig. 4 a Linear regression model showing the predictor 'mean monthly dosage of SCIG 20\%'. Blue dots represent data observed. b Linear regression model showing the predictor 'mean monthly dosage of IgG per kilogram of body weight'. c Linear regression model showing the predictor 'BMI'. Red line represents the linear regression line. Light grey stripe: confidence region of the regression line. Blue dots: data observed. BMI body mass index, $S C I G$ subcutaneous immunoglobulin, $\operatorname{Ig} G$ immunoglobulin $\mathrm{G}$

non-obese patients, suggesting the similar bioavailability of administered immunoglobulin irrespective of BMI.
Additionally, in a cohort of 107 patients with common variable immunodeficiency, Khan and colleagues found no relationship between the annual dose of IgG and trough IgG levels regardless of infusion frequency or adjustment for weight or body mass index [21]. Conversely, a poster presented in 2017 by Checkley et al. [22] suggested that increasing the SCIG dose might improve clinical outcomes in PID patients suffering from frequent infections. On the other hand, we should consider that immunoglobulin does not distribute to adipose tissue and is only present in the intravascular space and extracellular fluids. Indeed, various authors [23,24] recently suggested the use of adjusted BMI instead of the actual BMI for obese patients because subjects with increased adipose tissue are supposed to have increased extracellular fluid compared with normalweight subjects.

In our analysis, the mean dosage of IgG per kilogram of body weight per month was $365.5 \mathrm{~g}$. Although this predictor was slightly below the minimum generally recommended by guidelines, the serum levels remained stable. In fact, in all patients included in the pooled database, the mean trough concentration was above the threshold considered protective against most infections.

This finding supports the need for individualization of the posology according to patient clinical conditions and $\mathrm{IgG}$ levels. Tailoring the IgG dosage based on the serum IgG levels of the patient has also been suggested by other studies [25]. While deciding the dosing interval, even the preferences of the patient may be taken into account. In fact, by using a pharmacokinetic model and simulation, Landersdorfer et al. [26] have reported that it is possible to administer the same total IgG dose as one biweekly dose (once every 2 weeks, such as in the IBIS cohort) or two weekly doses in SCIG therapy without any changes in the IgG peaks and trough concentrations (only a $\pm 10 \%$ variability is observed during the 14-day period).

This study has some limitations. First, the two patient populations were significantly different in terms of age; IBIS patients were almost 8 years younger compared with those analyzed in the SHIFT study. Second, a difference in mean monthly IgG dosage was observed when the pediatric population was stratified according to weight categories, possibly due to the low sample size in the underweight category (five patients). Third, trough-level information was not available for all patients; however, the availability of these data for approximately $80 \%$ of patients may represent a reliable overview.

In addition, it should be considered that the trough level observed during IVIG treatment represents the minimum level attained at the end of the interval between two consecutive infusions, whereas the trough level detected during SCIG therapy represents an estimate that should not differ by more than $10 \%$ from the mean level during 
the therapy and is frequently defined as 'the steady-state level'. This consideration may modify the interpretation of pre-infusion IgG levels during IVIG or SCIG. Nevertheless, in the European Union, the monthly dose of IVIG is maintained when switching to SCIG, whereas a conversion factor is applied in the United States.

It should also be considered that, by developing a pharmacokinetic model, Bexon and colleagues [27] have found that there is great pharmacokinetic variability among individuals due to several factors, and such variability may affect an individual IgG trough/dose relationship.

\section{Conclusions}

This analysis showed total monthly IgG dosages had comparable trends among weight categories (except for underweight patients), the mean pre-infusion trough concentration was comparable among the weight categories, and the only two independent predictors associated with pre-infusion IgG trough levels in patients previously treated with IVIG or SCIG $16 \%$ and then received SCIG $20 \%$ were the mean monthly dosage of SCIG $20 \%$ and the mean monthly dosage of $\mathrm{IgG}$ per kilogram of body weight. As highlighted by stepwise regression, BMI did not correlate with pre-infusion trough concentrations. All patients treated with SCIG $20 \%$ maintained a pre-infusion trough concentration greater than the recommended thresholds. This effect was obtained using a mean monthly dosage of $23 \mathrm{~g}$ (interquartile range 16-26), which was not differentiated on a BMI basis. Therefore, dose adjustment may not be necessary in overweight and obese patients.

Acknowledgements The authors acknowledge the other principal investigators of the SHIFT/IBIS studies: Carlo Agostini, Chiara Azzari, Maria Giovanna Danieli, Marco De Carli, Mario Di Gioacchino, Andrea Finocchi, Silvana Martino, Baldassarre Martire, Andrea Matucci, Viviana Moschese, Claudio Pignata, Alessandro Plebani, Isabella Quinti, Antonino Trizzino and Angelo Vacca. The authors also acknowledge Laura Fascio Pecetto from SEEd Medical Publishers for publishing support and journal styling services, and Massimiliano Povero from AdRes Health Economics and Outcomes Research for statistical analyses.

Dataset Availability Statement CSL will only consider requests to share individual patient data (IPD) that are received from systematic review groups or bona fide researchers. CSL will not process or act on IPD requests until 12 months after article publication on a public website. An IPD request will not be considered by CSL unless the proposed research question seeks to answer a significant and unknown medical science or patient care question. Applicable country-specific privacy and other laws and regulations will be considered and may prevent sharing of IPD. Requests for use of the IPD will be reviewed by an internal CSL review committee. If the request is approved and the researcher agrees to the applicable terms and conditions in a data sharing agreement, IPD that has been appropriately anonymized will be made available. Supporting documents, including study protocol and statistical analysis plan, will also be provided. For information on the process and requirements for submitting a voluntary data sharing request for IPD, please contact CSL at clinicaltrials@ cslbehring.com.

\section{Compliance with Ethical Standards}

Funding CSL Behring Italy sponsored and funded both the SHIFT (IgPro20_5001) and IBIS (IgPro20_5002) studies. Open Access publication of this article was funded by CSL Behring, Italy. Publishing support and journal styling services for this study were provided by SEEd Medical Publishers and funded by CSL Behring, Italy. Statistical analyses for this study were performed by AdRes Health Economics and Outcomes Research and funded by CSL Behring, Italy.

Conflict of interest AP and GS received an unrestricted grant from CSL Behring for their research activity; AV has received honoraria for lectures from CSL Behring; and GMB is an employee of CSL Behring. SR declares he has no conflicts of interest.

Research involving human participants All procedures performed in studies involving human participants were in accordance with the ethical standards of the institutional and/or national research committee and with the 1964 Helsinki declaration and its later amendments or comparable ethical standards.

Informed consent Informed consent was obtained from all individual participants included in the study, as specified in the SHIFT and IBIS studies.

Open Access This article is licensed under a Creative Commons Attribution-NonCommercial 4.0 International License, which permits any non-commercial use, sharing, adaptation, distribution and reproduction in any medium or format, as long as you give appropriate credit to the original author(s) and the source, provide a link to the Creative Commons licence, and indicate if changes were made. The images or other third party material in this article are included in the article's Creative Commons licence, unless indicated otherwise in a credit line to the material. If material is not included in the article's Creative Commons licence and your intended use is not permitted by statutory regulation or exceeds the permitted use, you will need to obtain permission directly from the copyright holder.To view a copy of this licence, visit http://creativecommons.org/licenses/by-nc/4.0/.

\section{References}

1. Lim MS, Elenitoba-Johnson KS. The molecular pathology of primary immunodeficiencies. J Mol Diagn. 2004;6(2):59-83. https ://doi.org/10.1016/S1525-1578(10)60493-X.

2. Picard C, Bobby Gaspar H, Al-Herz W, et al. International union of immunological societies: 2017 primary immunodeficiency diseases committee report on inborn errors of immunity. J Clin Immunol. 2018;38(1):96-128. https://doi.org/10.1007/s 1087 5-017-0464-9.

3. Bousfiha AA, Jeddane L, Ailal F, et al. Primary immunodeficiency diseases worldwide: more common than generally thought. J Clin Immunol. 2013;33(1):1-7. https://doi.org/10.1007/s1087 5-012-9751-7.

4. ESID database statistics. Registry Working Party ESID Database Statistics. Major immunodeficiency groups. https://esid.org/Worki 
ng-Parties/Registry-Working-Party/ESID-Database-Statistics. Accessed 16 Apr 2019

5. Pecoraro A, Crescenzi L, Granata F, et al. Immunoglobulin replacement therapy in primary and secondary antibody deficiency: the correct clinical approach. Int Immunopharmacol. 2017;52:136-214. https://doi.org/10.1016/j.intimp.2017.09.005.

6. Chapel H, Gardulf A. Subcutaneous immunoglobulin replacement therapy: the European experience. Curr Opin Allergy Clin Immunol. 2013;13(6):623-9. https://doi.org/10.1097/ACI.0000000000 000013.

7. Bonilla FA, Barlan I, Chapel H, et al. International consensus document (ICON): common variable immunodeficiency disorders. J Allergy Clin Immunol Pract. 2016;4(1):38-59. https://doi. org/10.1016/j.jaip.2015.07.025.

8. Canessa C, Iacopelli J, Pecoraro A, et al. Shift from intravenous or $16 \%$ subcutaneous replacement therapy to $20 \%$ subcutaneous immunoglobulin in patients with primary antibody deficiencies. Int J Immunopathol Pharmacol. 2017;30(1):73-82. https://doi. org/10.1177/0394632016681577.

9. Vultaggio A, Azzari C, Ricci S, et al. Biweekly Hizentra ${ }^{\circledR}$ in primary immunodeficiency: a multicenter, observational cohort study (IBIS). J Clin Immunol. 2018;38(5):602-9. https://doi. org/10.1007/s10875-018-0528-5.

10. World Health Organization. Regional Office for Europe. Body mass index-BMI. http://www.euro.who.int/en/health-topics/ disease-prevention/nutrition/a-healthy-lifestyle/body-mass-index -bmi. Accessed 7 Mar 2019.

11. World Health Organization. Growth reference 5-19 years. BMIfor-age (5-19 years). https://www.who.int/growthref/who20 07_bmi_for_age/en/. Accessed 7 Mar 2019.

12. Cacciari E, Milani S, Balsamo A, et al. Italian cross-sectional growth charts for height, weight and BMI (2-20 yr). J Endocrinol Invest. 2006;29(7):581-93.

13. R Core Team. R: a language and environment for statistical computing. R Foundation for Statistical Computing, Vienna, Austria. 2014. http://www.R-project.org/. Accessed 8 Mar 2019.

14. Istituto Nazionale di Statistica (ISTAT). Infografiche. Se fossimo in 100: infografica. 29 dicembre 2016. Available at: https://www. istat.it/it/archivio/196779. Accessed 8 Mar 2019.

15. NCD Risk Factor Collaboration (NCD-RisC). Worldwide trends in body-mass index, underweight, overweight, and obesity from 975 to 2016: a pooled analysis of 2416 population-based measurement studies in 128.9 million children, adolescents, and adults. Lancet. 2017;1:4. https://doi.org/10.1016/s0140-6736(17)32129-3 (Epub 10 Oct 2017).

16. Borte M, Kriván G, Derfalvi B, et al. Efficacy, safety, tolerability and pharmacokinetics of a novel human immune globulin subcutaneous, 20\%: a Phase 2/3 study in Europe in patients with primary immunodeficiencies. Clin Exp Immunol. 2017;187(1):146-59. https://doi.org/10.1111/cei.12866.
17. Spadaro G, Vultaggio A, Alberto Bosi A, et al. Rapid infusions of human normal immunoglobulin $50 \mathrm{~g} / \mathrm{l}$ are safe and well tolerated in immunodeficiencies and immune thrombocytopenia. Int Immunopharmacol. 2017;44:38-42. https://doi.org/10.1016/j. intimp.2016.12.030.

18. Simoens S. Treatment of primary immunodeficiency with Kiovig: a literature review. Adv Ther. 2010;27(3):142-9. https://doi. org/10.1007/s12325-010-0016-7.

19. Bezrodnik L, Gómez Raccio A, Belardinelli G, et al. Comparative study of subcutaneous versus intravenous IgG replacement therapy in pediatric patients with primary immunodeficiency diseases: a multicenter study in Argentina. J Clin Immunol. 2013;33(7):1216-22. https://doi.org/10.1007/s10875-013-9916-z.

20. Shapiro R. Subcutaneous immunoglobulin (16 or 20\%) therapy in obese patients with primary immunodeficiency: a retrospective analysis of administration by infusion pump or subcutaneous rapid push. Clin Exp Immunol. 2013;173(2):365-71. https://doi. org/10.1111/cei.12099.

21. Khan S, Grimbacher B, Boecking C, et al. Serum trough IgG level and annual intravenous immunoglobulin dose are not related to body size in patients on regular replacement therapy. Drug Metab Lett. 2011;5(2):132-8. https://doi.org/10.2174/187231211795305 302.

22. Checkley A, Kristofek L, Kile S, et al. Optimizing subcutaneous dosing of immunoglobulin in obese primary immune deficiency patients treated in the home. Ann Allergy Asthma Immunol. 2017;119(5):S65. https://doi.org/10.1016/j.anai.2017.08.175.

23. Ameratunga R. Initial intravenous immunoglobulin doses should be based on adjusted body weight in obese patients with primary immunodeficiency disorders. Allergy Asthma Clin Immunol. 2017;13:47. https://doi.org/10.1186/s13223-017-0220-y.

24. Hodkinson JP. Considerations for dosing immunoglobulin in obese patients. Clin Exp Immunol. 2017;188(3):353-62. https:// doi.org/10.1111/cei.12955.

25. Berger M, Rojavin M, Kiessling P, et al. Pharmacokinetics of subcutaneous immunoglobulin and their use in dosing of replacement therapy in patients with primary immunodeficiencies. Clin Immunol. 2011;139(2):133-41. https://doi.org/10.1016/j. clim.2011.01.006

26. Landersdorfer CB, Bexon M, Edelman J, et al. Pharmacokinetic modeling and simulation of biweekly subcutaneous immunoglobulin dosing in primary immunodeficiency. Postgrad Med. 2013;125(6):53-61. https://doi.org/10.3810/pgm.2013.11.2712.

27. Bexon M, Edelman JM, Rojavin M, et al. Individuals, subgroups or cohorts? A population pharmacokinetic modeling approach to optimize dosing of Hizentra ${ }^{\circledR}$. J Allergy Clin Immunol Pract. 2013;131(2 Suppl):AB227. https://doi.org/10.1016/j. jaci.2012.12.1476. 